\title{
Highly Efficient Inverted Organic Photovoltaics Containing Aliphatic Hyperbranched Polymers as Cathode Modified Layers
}

Tzong-Yuan Juang ${ }^{a}$, Yu-Chi Hsu ${ }^{b}$, Bing-Huang Jiang ${ }^{b}$, Chih-Ping Chen ${ }^{b^{*}}$

${ }^{a}$ Department of Cosmeceutics, China Medical University, Taichung, Taiwan

${ }^{b}$ Department of Materials Engineering, Ming Chi University of Technology, New Taipei City, 24301, Taiwan 
a)

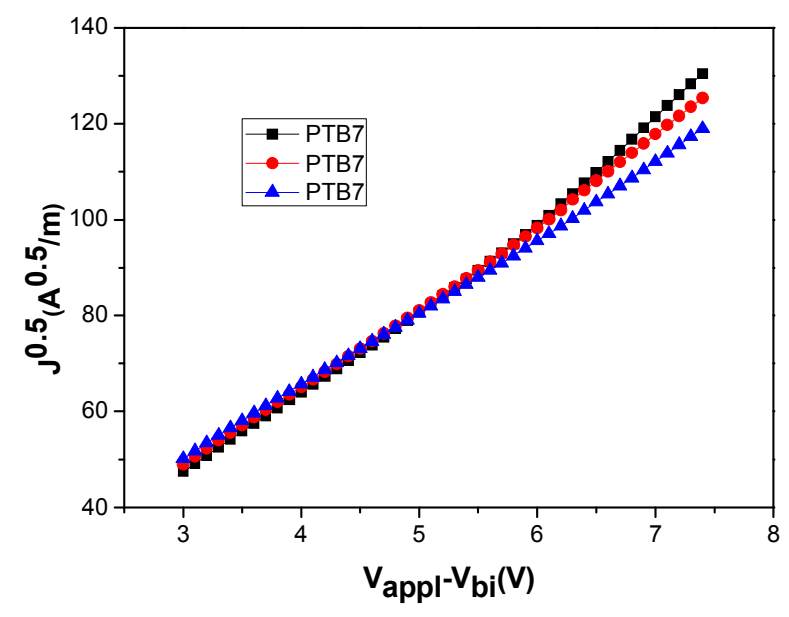

c)

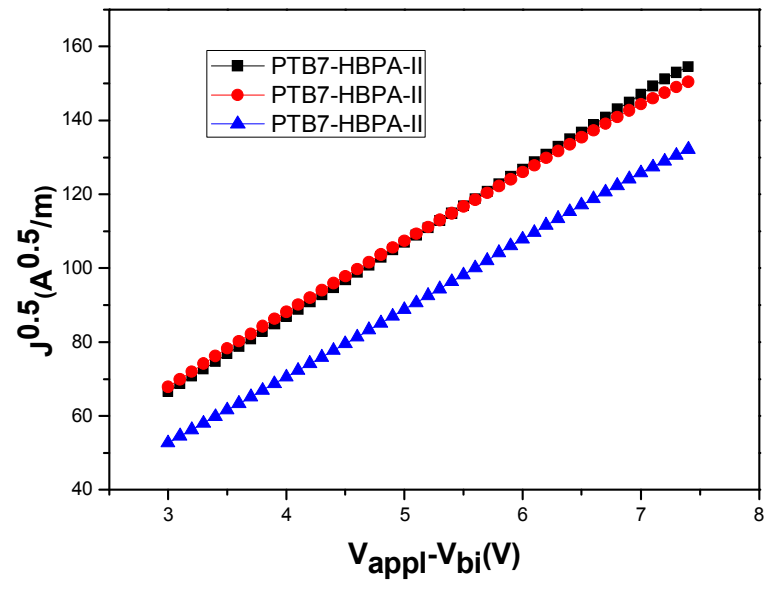

b)

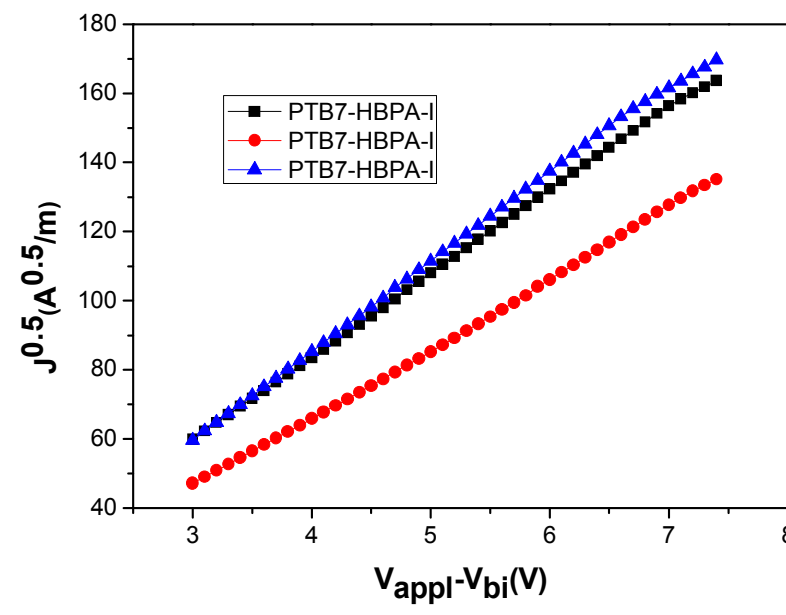

d)

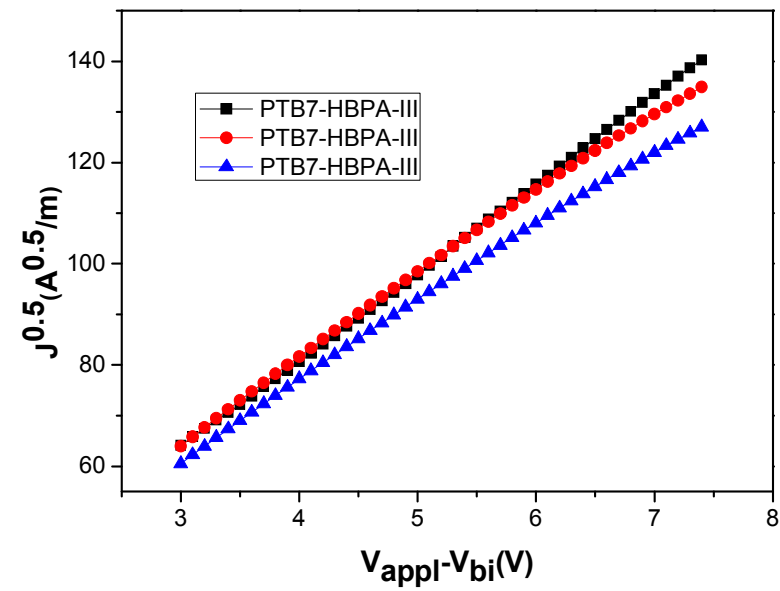

Figure S1. $J^{0.5}-V$ plots for electron-only devices a) PTB7, b) PTB7-HBPA-I, c) PTB7-HBPA-II, and d) PTB7-HBPA-III. 